\title{
Recent Advances of Non-Invasive MR pH Imaging Using Chemical Exchange Saturation Transfer
}

\author{
Wu RH* \\ Department of Medical Imaging, The $2^{\text {nd }}$ Affiliated \\ Hospital, Shantou University Medical College, Shantou, \\ China \\ *Corresponding author: Wu RH, Department of \\ Medical Imaging, Shantou University Medical College, \\ Shantou, China
}

Received: August 16, 2018; Accepted: September 18, 2018; Published: September 25, 2018

\section{Keywords}

Chemical Exchange Saturation Transfer (CEST); PH imaging; Magnetic Resonance (MR)

\section{Mini Review}

Alteration in tissue $\mathrm{pH}$ is an indicator of many pathological processes. Noninvasive MR $\mathrm{pH}$ imaging will be much helpful for disorder characterization and therapy, even monitoring cell viability. Noninvasive brain $\mathrm{pH}$ measurements have routinely relied on ${ }^{31} \mathrm{P}$ magnetic resonance spectroscopy techniques, which require additional hardware, take too long to be clinically useful, and provide very limited spatial resolution. Chemical Exchange Saturation Transfer (CEST) is a versatile technique for MR molecular imaging. The potential of Amide Proton Transfer (APT) had been proved for imaging $\mathrm{pH}$ effects in ischemic rat brain noninvasively [1].

With combination of amide and guanidyl CEST, the sensitivity of $\mathrm{pH}$-weighted $\mathrm{MR}$ imaging can be enhanced for ischemic rat brain in a recent study [2]. Iopamidol, as a chemical exchangedependent saturation transfer contrast medium, has been used to measure extracellular $\mathrm{pH}(\mathrm{pHe})$ [3]. A single dose of cariporide can induce a rapid change of intracellular $\mathrm{pH}(\mathrm{pHi})$ in animal glioblastoma multiforme, which is observed by using Amine/Amide Concentration- Independent Detection (AACID) CEST MR imaging
[4]. The major contributors to in vivo $\mathrm{T}_{1}$-normalized $\mathrm{MTR}_{\text {asym }}(3.5$ $\mathrm{ppm}$ ) contrast between white and gray matter in normal brain are $\mathrm{pH}$-insensitive macromolecular Magnetization Transfer (MT) and nuclear Overhauser enhancement. The $\mathrm{pH}$-sensitive amine and amide effects account for nearly $60 \%$ and $80 \%$ of the $\mathrm{MTR}_{\text {asym }}$ changes seen in white and gray matter, respectively, after global ischemia, indicating that $\mathrm{MTR}_{\text {asym }}$ is predominantly $\mathrm{pH}$-sensitive [5]. Clinical translation of $\mathrm{pH}$-weighted $\mathrm{MR}$ imaging has been conducted for diagnosing human brain tumors [6].

In our most recent studies, in vivo gas challenge in an experimental glioma model of rats showed that enhanced $\mathrm{pH}$-weighted MR imaging could more effectively localize tumor periphery. In addition, ioversol (a clinical CT contrast medium) CEST MR imaging can be exploited to achieve pHe mapping of human liver cancer microenvironment.

\section{References}

1. Zhou J, Payen JF, Wilson DA, Traystman RJ, van Zijl PC. Using the amide proton signals of intracellular proteins and peptides to detect $\mathrm{pH}$ effects in MRI. Nat Med. 2003; 9: 1085-1090.

2. Jin T, Wang P, Hitchens TK, Kim SG. Enhancing sensitivity of pH-weighted MRI with combination of amide and guanidyl CEST. Neuroimage. 2017; 157: 341-350.

3. Sun PZ, Longo DL, Hu W, Xiao G, Wu R. Quantification of iopamidol multi-site chemical exchange properties for ratiometric Chemical Exchange Saturation Transfer (CEST) imaging of pH. Phys Med Biol. 2014; 59: 4493-4504.

4. Albatany M, Li A, Meakin S, Bartha R. In vivo detection of acute intracellular acidification in glioblastoma multiforme following a single dose of cariporide. Int J Clin Oncol. 2018.

5. Zhou IY, Lu D, Ji Y, Wu L, Wang E, Cheung JS, et al. Determination of multipool contributions to endogenous amide proton transfer effects in global ischemia with high spectral resolution in vivo chemical exchange saturation transfer MRI. Magn Reson Med. 2018.

6. Shen Y, Xiao G, Shen Z, Zhang X, Tang X, Hu W, et al. Imaging of nuclear Overhauser enhancement at 7 and 3 T. NMR Biomed. 2017; 30 